\title{
A Situação do ensino da facoemulsificação no Brasil
}

\begin{abstract}
A cirurgia de catarata é um dos procedimentos cirúrgicos mais realizados no mundo. Atualmente, a técnica mais utilizada nas cirurgias de catarata é a facoemulsificação (Faco), em razão da possibi lidade de rápida recuperação visual e ao reduzido índice de complicações intraoperatórias ${ }^{(1,2)}$. Complicações durante a cirurgia de catarata são raras, porém representam significativo problema econômico e clínico para o paciente ${ }^{(3)}$. Assim, o ensino da Faco, mantendo-se o elevado padrão de satisfação dos pacientes, é um grande desafio para os centros de treinamento.

Com o objetivo de formar mais cirurgiões habilitados, e incentivados pela necessidade e pelo estímulo à realização de maior quantidade de cirurgias de catarata no Brasil, a partir de 2001, muitos hospitais universitários elevaram o número de médicos residentes e aumentaram a capacidade dos centros cirúrgicos para a realização de cirurgias pela técnica de $\mathrm{Faco}^{(3,4)}$. Seguindo esta tendência, o Conselho Brasileiro de Oftalmologia (CBO) criou, em 2008, uma comissão para facilitar a aquisição de modernos aparelhos de facoemulsificação por hospitais escolas. O resultado destas medidas é que, atualmente, a maioria dos serviços de residência no Brasil ensinam a técnica de Faco.
\end{abstract}

\section{Tabela 1}

\section{Indicadores quantitativos e qualitativos médios, de cada residente ao final do programa, relacionados ao ensino de facoemulsificação em hospitais escolas do Brasil (2011)}

Número de cirurgias por Faco realizadas
Quantidade de horas de aulas teóricas
Quantidade de horas de Wet Lab
Tipo de facoemulsificador
Orientação de um cirurgião experiente

130 Facos

Menos de 10 horas

Menos de 6 horas

Moderno (preciso e seguro)

inião experiente

Sim

* Hospitais escolas avaliados: USP-SP; USP-RP; UNESP; FM do ABC; UFRJ; HG de Bom Sucesso; HSE do RJ; UFMG; UFPR; H Evangélico de POA; Sta Casa de POA

Com o intuito de avaliar a qualidade do ensino da Faco nos hospitais escolas, entramos em contato com preceptores de 11 (onze) centros formadores de residentes, em SP, RJ, MG, RS e PR, para obter as informações relacionadas na Tabela 1 , as quais iremos discutir.

Constatamos que, na média dos hospitais pesquisados, a quantidade de Facos realizadas pelos residentes, ao final do programa, foi de aproximadamente 130 cirurgias, variando de 80 a 400 procedimentos. Baseado em nossa experiência, ao longo de treze anos treinando cirurgiões, consideramos esta uma quantidade suficiente para que o jovem oftalmologista ingresse no mercado privado, com competência para realizar cirurgias pela técnica da Faco. Esta percepção é confirmada por Taravella et al., em um estudo prospectivo com 324 casos operados por residentes, que observaram que o cirurgião em treinamento adquire competência para operar com índice de complicações e com tempo de cirurgia comparável a cirurgiões experientes após ter realizado mais de $75 \mathrm{Facos}$, e quando realiza mais de 100 procedimentos, adquire maior precisão, principalmente para a fratura e para conquista do núcleo ${ }^{(5)}$.

Em seguida, avaliamos indicadores da qualidade do treinamento cirúrgico e observamos que todos os serviços pesquisados dispunham de facoemulsificadores modernos (seguros e precisos), e que a grande maioria das cirurgias era realizada sob orientação de um cirurgião experiente, dois pré-requisitos que 
consideramos fundamentais para o ensino adequado da técnica. Em 2010, publicamos um estudo em que constatamos que a Faco realizada por cirurgiões em treinamento sob supervisão apresentava o mesmo índice de complicações de quando realizada por cirurgiões experientes, corroborando a hipótese de que um residente com orientação adequada é capaz de operar com segurança ${ }^{(6)}$.

Assim, o ensino de facoemulsificação com qualidade exige um centro cirúrgico devidamente equipado e com disponibilidade de cirurgiões capacitados para supervisionar o treinamento, realidade que está presente nos principais centros de ensino do Brasil. Todavia, quando pesquisamos outro importante indicador de qualidade no ensino cirúrgico, que é a carga teórica oferecida, o resultado foi surpreendente. Constatamos que, a maioria dos centros de ensino oferecem menos do que 10 horas de teoria específica de Faco.

Em nossa experiência ensinando cirurgiões, percebemos que o refinamento e aperfeiçoamento na técnica da Faco envolve o conhecimento do aparelho de facoemulsificação, com suas particularidades físicas e fluídicas, e das forças mecânicas associadas às manobras cirúrgicas. A correta programação e o controle das funções do facoemulsificador são fundamentais para a realização de uma cirurgia precisa e segura. A escolha criteriosa da técnica de fratura do núcleo, assim como a compreensão das manobras envolvidas em sua realização, são essenciais para uma estratégia cirúrgica eficaz e reprodutível. Informações relativas aos movimentos e vetores associados à capsulorrexe são de grande importância para o controle da excursão do flap.

Muitas destas valiosas informações não estão disponíveis em livros textos, ou podem não ser bem compreendidas sem auxílio de recursos didáticos especiais, como vídeos e animações. A compreensão destes conceitos é importante para melhorar a performance durante o aprendizado e, posteriormente, aperfeiçoar a técnica cirúrgica, agregando precisão e segurança ao procedimento. As etapas mais difíceis para o cirurgião iniciante (incisão e capsulorrexe), assim como as etapas mais difíceis para o cirurgião em fase avançada de treinamento (sulco, fratura e conquista) podem ser facilitadas com o adequado conhecimento teórico dos princípios envolvidos nestas manobras. O que aprimoraria e tornaria mais seguro o treinamento.

A Faco deve ser percebida como é, uma cirurgia diferente da extração extracapsular (EECP.). A necessidade de se fraturar o núcleo do cristalino para, em seguida, fragmentá-lo e aspirá-lo dentro do saco capsular, sob a ação de um aparelho de facoemulsificação, exige uma estratégia cirúrgica que não pode ser treinada durante a EECP e, portanto, deve ser bem compreendida antes de se operar olhos humanos. Talvez, um grande equívoco durante o aprendizado da Faco seja considerar que, a experiência prévia na técnica da EECP poderá ser muito útil, pois em verdade, pouco desta experiência é aproveitada. Desta forma, acreditamos que o primeiro estágio do treinamento cirúrgico da Faco seja estudar cuidadosamente a nova técnica, suas etapas, particularidades, principais dificuldades e complicações, bem como os princípios físicos e fluídicos do facoemulsificador, para em seguida, com auxílio de um cirurgião experiente, começar as primeiras cirurgias em humanos. E não considerar que apenas o domínio da técnica de EECP seja suficiente para capacitar o cirurgião a realizar a Faco.

Woodfield et al., observaram que o índice de complicações cirúrgicas durante o treinamento de residentes do segundo e do terceiro ano na Faco era semelhante, concluindo que a Faco poderia ser ensinada de maneira segura a partir do segundo ano de residência ${ }^{7}$. Em centros de excelência em ensino como o Johns Hopkins University, o residente já inicia o treinamento em Faco no primeiro ano, sempre com casos selecionados e sob orientação adequada ${ }^{(7)}$. Concordamos com esta posição, principalmente ao considerar que quanto mais cedo o residente iniciar o treinamento na Faco, mais tempo ele terá para estudar os princípios teóricos envolvidos na técnica, compreendendo melhor as cirurgias que observar e as aulas em congressos e cursos frequentados. O residente de primeiro e segundo ano possui, provavelmente, mais condições de estudar a teoria do que o de terceiro ano, em que o foco, em geral, é o de efetivamente realizar a cirurgia.

A Faco não é uma técnica em que somente a habilidade cirúrgica é importante. Tão importante quanto a "mão cirúrgica", é o conhecimento da estratégia de fratura do núcleo cristaliniano e dos recursos e parâmetros do aparelho de facoemulsificação. A boa notícia é que estes últimos requisitos podem ser adquiridos fora do centro cirúrgico, por meio da literatura e, principalmente, em cursos específicos de imersão. Em 2008, publicamos um estudo que demonstrou a necessidade e a eficácia de cursos intensivos, 
de curta duração, sobre a transferência das habilidades propostas, como complementação aos grandes congressos gerais ou de especialidade ${ }^{(8)}$.

Com relação ao Wet Lab (laboratório do treinamento), limitações financeiras e logísticas têm restringido progressivamente sua aplicação. No entanto, após 13 anos de experiência no ensino da Faco, percebemos que com o conhecimento adequado da técnica cirúrgica e dos parâmetros/recursos do facoemulsificador, a necessidade do Wet Lab passa a não ser fundamental, principalmente ao se considerar que a tendência mundial seja de se realizar treinamento experimental com programas computadorizados de simulação cirúrgica (Dry labs), onde etapas como a incisão e a capsulorrexe são mais realistas ${ }^{(9)}$.

Assim, consideramos que a situação atual do ensino da Faco no Brasil seja adequada com relação à oferta de cirurgias para treinamento, porém em relação à qualidade do treinamento, embora a maioria dos hospitais escolas disponham de aparelhos seguros e orientação adequada, ainda falta maior investimento na oferta de informações teóricas, e melhor discussão com os preceptores dos centros de treinamento, sobre qual seria a época apropriada para o início do aprendizado cirúrgico. Acreditamos que quanto mais cedo se iniciar o treinamento na Faco, mais tempo, condições e motivação o residente terá para se capacitar na teoria envolvida e, consequentemente, melhor seria o aproveitamento de cada cirurgia realizada.

Newton Kara-Junior

Professor colaborador livre-docente Faculdade de Medicina da Universidade de São Paulo - USP - São Paulo (SP), Brasil

\section{ReFERÊNCIAS}

1. Leaming DV. Practice styles and preferences of ASCRS members-1998 survey. J Cataract Refract Surg.1999;25(6):851-9.

2. Kara-Junior, N Sirtoli MG, Santhiago MR, Parede TR, Espíndola RF, Carvalho R de S,. versus extracapsular extraction: governmental costs. Clinics (São Paulo). 2010;65(4):357-61.

3. Kara-Junior N. Importância do centro cirúrgico ambulatorial para realização de cirurgias de catarata em larga escala. Rev Bras Oftalmol. 2011;70(2):75-6.

4. Kara-Junior N, Parede TR, Santiago MR, Espindola RF, Mazurek MG, Carvalho RS. Custo social de duas técnicas de cirurgia de catarata no Brasil. Rev Saúde Pública. 2010;44(5):957-62.

5. Taravella MJ, Davidson R, Erlanger M, Guiton G, Gregory D. Characterizing the learning curve in phacoemulsification. J Cataract Refract Surg. 2011;37(6):1069-75.
6. Barreto Junior J, Primiano Junior H, Espíndola RF, Germano RAS, Kara-Junior N. Cirurgia de catarata realizada por residentes: avaliação dos riscos. Rev Bras Oftalmol. 2010;69(5):301-5.

7. Woodfield AS, Gower EW, Cassard S, Ramanthan S. Itraoperative phacoemulsification complication rates of second and third year ophthalmology residents. Ophthalmology. 2011;118(5):954-8.

8. Kara-Jose, Noma R, Carvalho RS, Chinaglia MZ, Kara-Junior N. Investimento e satisfação em curso de curta duração: modelo de avaliação. Rev Bras Oftalmol. 2008;67(1):7-11.

9. Prakash G, Jhanji V, sharma N. Assessment of perceived difficultes by residents in performing routine steps inphacomulsification surgery and in managing complications. Can J Ophthalmol. 2009;44(3):284-7 